\title{
Extração de informações da cena urbana combinando imagens RADARSAT-2 e GeoEye
}

Anna Carolina Cavalheiro ${ }^{1}$

\section{Resumo}

Neste trabalho, foi analisada a contribuição da imagem RADARSAT-2 na discriminação de elementos de uma cena urbana, quando fundida com uma imagem GeoEye. A vantagem da imagem-radar é que sua tomada independe das condições atmosféricas, e a resolução espacial nominal no modo ultrafino é de 3 metros. Já as imagens multiespectrais, mesmo sendo de alta resolução, não permitem discriminar todos os elementos que compõem uma cena urbana. O objetivo deste trabalho foi discriminar elementos da cena, utilizando a combinação de informações obtidas de imagem óptica e de radar, e também verificar se o modelo de transformação projetiva é adequado para a correção geométrica da imagem-radar. Pretende-se, ainda, analisar o efeito dos filtros para redução do speckle na imagem e constatar a contribuição da imagem radar para a identificação de elementos presentes na cena. A transformação projetiva apresentou um resultado satisfatório na correção da imagem, apesar de o ângulo de visada ser lateral; os valores de resíduos para os pontos de controle e verificação ficaram menores que 1 metro. Para tratar o speckle, verificou-se que o filtro estatístico de média com janela $5 \times 5$ foi o que permitiu sua eliminação com maior eficiência. Os resultados dos experimentos de classificação, utilizando árvore de decisão, apresentaram-se coerentes com a realidade da área de estudo. $O$ melhor resultado foi verificado no experimento utilizando a imagem GeoEye segmentada, combinada com a imagem-radar, uma vez que essa contribui para a caracterização de estruturas metálicas que apresentam alto fator de reflexão. Foi possível comprovar a confiabilidade da técnica na visita de campo.

Palavras-chave: GeoEye. Speckle. Imagem-radar. Classificação de imagens.

\section{Introdução}

As imagens orbitais geradas por sistemas ativos têm sido objeto de estudo de pesquisas aplicadas a diversos fenômenos. O sistema Radio Detection and Ranging (RADAR) é um desses sistemas que geram imagens utilizando energia eletromagnética na faixa de micro-ondas.

Um exemplo de sensor radar é o RADARSAT-2, lançado em 2007, pela Canadian Space Agency (CSA). Esse sensor possui resolução espacial nominal de 3 metros, com direção de visada à esquerda ou à direita, e receptores GPS a bordo. Além disso, é possível programar a aquisição de imagens selecionando o valor da frequência, o ângulo de incidência e a polarização, de acordo com o enfoque do estudo a ser realizado, conforme o Canada Centre For Remote Sensing (CCRS). O uso de sensores como o RADARSAT-2 para aquisição de imagens proporciona uma grande vantagem em relação aos sensores ópticos, pois independe das condições atmosféricas.

Técnicas de Processamento Digital de Imagens (PDI) devem ser aplicadas na preparação da imagem SAR, Synthetic Aperture Radar, para que essa se torne um produto cartográfico confiável. As principais etapas desse processamento são: a filtragem da imagem para eliminação de ruídos e a cor-

1 Universidade Federal do Paraná. annaufpr@gmail.com 
reção geométrica. Mesmo após o processamento, as imagens radar são de complexa interpretação, pois não há uma cognição visual intuitiva como numa imagem óptica.

A classificação de imagens multiespectrais, mesmo quando de alta resolução, pode apresentar confusão entre as classes, em decorrência da diversidade de materiais existentes numa cena urbana. Outras faixas de imageamento, como a do infravermelho médio, por exemplo, poderiam auxiliar a discriminação dos elementos, mas não são adquiridas com a mesma resolução. Os dados obtidos de uma imagem-radar fornecem informação relativa à orientação das vias, rugosidade da superfície e geometria dos objetos imageados, e podem ser obtidos em alta resolução. A combinação da imagem multiespectral com a imagem radar pode resultar em uma fonte de informações mais completa, permitindo, assim, um estudo mais aprofundado da região de interesse.

O objetivo geral desta pesquisa foi estudar a viabilidade de combinação de imagens ópticas com imagens-radar para discriminar elementos presentes numa cena urbana. Certos cuidados como a calibração, a filtragem e a compatibilização geométrica dos dados foram etapas imprescindíveis para não comprometer as análises.

Um dos objetivos específicos foi verificar se o modelo de transformação projetiva é adequado para a correção geométrica da imagem RADAR. A correção geométrica realizada, utilizando o modelo de transformação projetiva, apresentou um resultado satisfatório na correção da imagem; apesar de o ângulo de visada ser lateral, os valores de resíduos para os pontos de controle e verificação ficaram menores que 1 metro.

\section{Fundamentos}

\section{Imagem RADARSAT}

Os sistemas imageadores de micro-ondas, chamados RADAR (Radio Detection and Ranging), consistem em um equipamento gerador de pulsos, um transmissor, um seletor que coordena a transmissão e a recepção da energia das micro-ondas, uma antena, um receptor e um equipamento de gravação (CCRS, 2008).

A maioria dos radares de sensoriamento remoto funciona em comprimentos de onda entre $0,5 \mathrm{~cm}$ e $75 \mathrm{~cm}$. A capacidade de penetração da onda através da chuva ou em uma camada superficial de um alvo aumenta quanto maior for o comprimento de onda. Os imageadores RADARSAT operam na chamada Banda C, que compreende a faixa de 3,75 a 7,5 cm (8 a $14 \mathrm{GHz}$ ).

O RADARSAT-1 foi lançado pela Agência Espacial Canadense em novembro de 1995, em uma órbita quase polar heliossíncrona, a $798 \mathrm{~km}$ da superfície da Terra. A órbita do satélite tem um ciclo de 24 dias, mas pode ser programado para um ciclo de revisita mais frequente. Esse sensor permite também customizar o modo de aquisição, dependendo da aplicação, do tipo de terreno, da necessidade de estereoscopia, da resolução espacial desejada e da frequência da área de cobertura. Para cada modo de aquisição da cena está disponível um determinado número de posições, com intervalos de ângulos de incidência diferentes (CCRS, 2008).

O RADARSAT-2, lançado em dezembro de 2007, foi projetado com avanços tecnológicos significativos, que incluem resolução ultrafina de 3 metros, direção de visada à esquerda ou à direita, possibilidade de seleção da polarização pelo usuário e receptores GPS a bordo. Oferece todos os modos de imageamento do RADARSAT-1, além de algumas capacidades adicionais.

Entre as inovações do RADARSAT-2, podemos citar os modos de imageamento com polarização paralela (HH e VV) e cruzada (HV e VH); o sensor também pode imagear à esquerda ou à direita 
do nadir. A presença de receptores GPS a bordo permite monitorar a posição do satélite e a retificação mais precisa das imagens.

\section{Imagens GeoEye}

O Satélite GeoEye foi lançado em 2008 pela base militar de Vanderberg, Califórnia (EUA). É atualmente o sistema de imageamento orbital com maior resolução espacial, sendo o valor nominal do pixel de 0,41 metros na imagem pancromática e 1,6 metros no modo multiespectral. A imagem multiespectral possui quatro bandas que abrangem as faixas do visível (RGB) e do infravermelho próximo (GEOEYE, 2010).

O satélite GeoEye possui órbita polar e tempo de revisita de três dias ou menos, dependendo do ângulo de visada utilizado. A altitude de voo é de $681 \mathrm{~km}$, com uma velocidade orbital de cerca de $16.800 \mathrm{~km} / \mathrm{h}$. Sua órbita heliossíncrona permite que ele passe sobre uma determinada área por volta das 10h30, hora local, a cada dia (GEOEYE, 2010).

A área imageada pode chegar a 350 quilômetros quadrados de terreno por dia. Os sensores foram desenvolvidos pela empresa GeoEye, especializada em tratamento de imagens digitais, e o satélite foi construído pelo programa NextView da Agência Geoespacial Nacional dos Estados Unidos (GEOEYE, 2010).

\section{Caracterização da cena urbana}

O mapeamento urbano com imagens radar pode ajudar a delimitar a distribuição de aglomerações urbanas e de elementos importantes para aplicações, como prevenção de desastres ou modelagem de impactos (MOLCH, 2009). Os dados SAR são diferentes dos dados de sensores ópticos, não só na forma como a informação é coletada, mas também na geometria de aquisição. A geometria da visada lateral introduz efeitos como a distorção de relevo, principalmente quando há muitas variações no terreno ou edificações altas.

As características da antena, o mecanismo do retroespalhamento e o processo de formação da imagem introduzem padrões a pixel que resultam na imagem SAR. Por essa razão, a imagem-radar não é comparável com a informação contida na imagem óptica. Métodos de classificação, baseados em similaridade radiométrica, utilizados em dados ópticos não são adequados aos dados-radar.

A representação de uma área urbana sofre influência dos parâmetros de formação da imagem, sendo eles:

1. Comprimentos de onda e polarização;

2. Geometria da aquisição, incluindo direção da órbita, ângulo de incidência local e posição do sensor;

3. Propriedades de alvos e vizinhança, incluindo rugosidade e espaçamento.

Os sensores ativos podem adquirir imagens tanto na órbita ascendente quanto na descendente e também no período da noite. O sensor RADARSAT-2 é programado para adquirir imagens tanto na visada à esquerda quanto à direita, e também se pode selecionar a resolução da aquisição (MOLCH 2009).

Nas imagens obtidas com sensor radar, as edificações geralmente apresentam alta resposta, ou seja, valor de pixel elevado, com exceção de áreas que não são alcançadas pelo sensor, como alguns cantos de muro, ou em materiais não refletivos como plástico e madeira. A vegetação tem forte influência na representação de áreas urbanas, visto que o sinal absorvido e refletido é afetado pelo volume do espalhamento provocado pela rugosidade do alvo e grau de atenuação do sinal. Assim, quanto 
maior o retroespalhamento do alvo, menor será o retorno ao sensor. Além disso, a resposta do alvo (valor numérico registrado pelo sensor) sofre influência da direção da órbita, do ângulo de incidência e da posição do alvo, em relação à direção de visada e do azimute.

\section{Materiais e métodos}

\section{Materiais}

Utilizou-se neste trabalho uma imagem RADARSAT-2 com polarização horizontal $(\mathrm{HH})$ e resolução espacial nominal de 3 metros. Essa imagem foi calibrada, filtrada e corrigida geometricamente, conforme os procedimentos descritos na sequência. Para correção geométrica, utilizou-se um Modelo Digital de Terreno gerado a partir de curvas de nível, fornecidas pelo IPPUC (Instituto de Pesquisa e Planejamento Urbano de Curitiba), e um mosaico de ortofotos georreferenciadas da área de estudo fornecido pelo mesmo Instituto.

Também foi utilizada uma imagem GeoEye com resolução espacial nominal de 0,5 metro, contendo as 3 bandas do visível e a do infravermelho próximo. Essa imagem foi registrada e reamostrada para a resolução de 2,5 metros para compatibilização com a imagem-radar.

A área de estudo abrange cerca de $36 \mathrm{~km}^{2}$ na latitude $25^{\circ} 25^{\prime} 48^{\prime \prime}$ S e longitude 49¹6'15" W, porção nordeste da cidade de Curitiba, capital do Estado do Paraná. Além das imagens, foram utilizados os softwares ArcGis, EDSON, ENVI, Matlab MultiSpec e Weka para o processamento. 


\section{Procedimentos metodológicos}

As etapas realizadas para desenvolvimento dos experimentos estão resumidas no fluxograma (Figura 1).

\section{PROCEDIMENTOS METODOLÓGICOS}

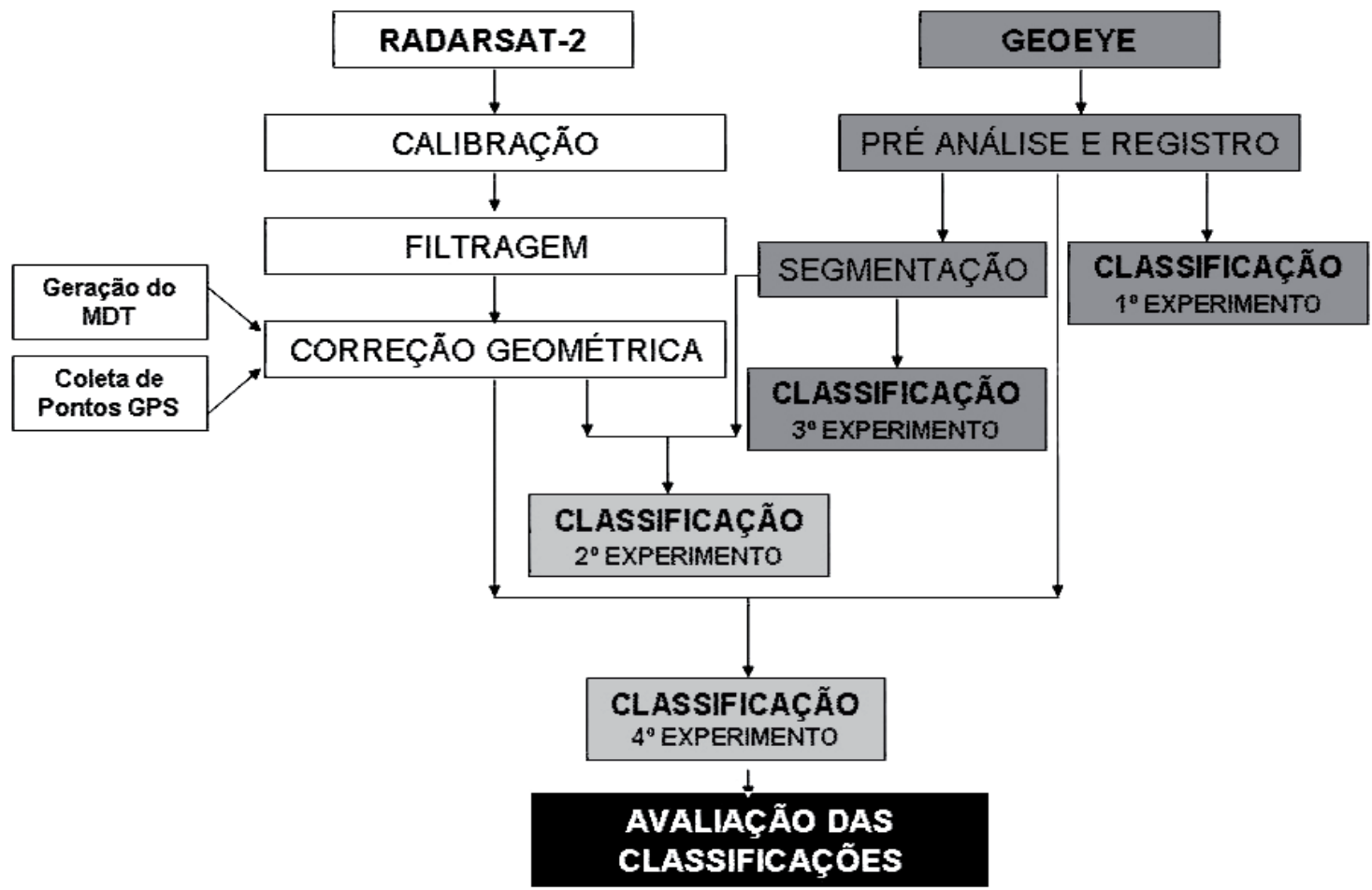

Figura 1. Fluxograma dos Procedimentos Metodológicos.

Fonte: Elaboração própria.

\section{Calibração}

A calibração de uma imagem-radar é um processo importante para garantir que os valores presentes na imagem tenham equivalência em uma escala numérica com unidade de medida em decibéis. $O$ resultado da calibração é uma imagem em escala de decibéis, e um bel corresponde a uma diferença unitária em logaritmos de base 10 entre dois números. Um decibel (dB) é 0.1 bel, isto é, uma diferença de 0.1 na base logarítmica 10 entre dois números (SOARES, 2010).

As imagens RADARSAT-2 vêm acompanhadas de uma tabela para escalonamento dos valores originais chamadas LUT file. Essa tabela fornece valores que permitem converter os valores digitais originais para a escala sigma-zero, aplicando uma constante de compensação da distância entre o sensor e o alvo.

Cada coluna da imagem possui um valor correspondente na tabela. Para se obter o valor calibrado da imagem, o valor original deve ser elevado ao quadrado, depois adicionado a uma constante (B), fornecida pelo fabricante, e dividido pelo valor do ganho (A) correspondente ao valor da coluna do pixel na tabela de correção, como demonstra a Equação 1.

$$
\text { Valor calibrado }=(\underline{\text { valor digital }})^{2} \underline{+B}
$$


O fator da correção aumenta de acordo com a distância entre o alvo e a antena.

\section{Filtros}

Os efeitos de speckle podem ser significativamente reduzidos pela utilização de filtros adequados, melhorando a resolução radiométrica.

Os filtros não adaptativos consideram os parâmetros do sinal de toda a imagem e não as propriedades locais do retroespalhamento do terreno ou a natureza do sensor, por essa razão não são apropriados para filtragens de sinais provenientes de alvos móveis.

Os filtros adaptativos tradicionais normalmente requerem o cálculo de médias locais e seus respectivos desvios-padrões (coeficiente de variação). Esses filtros produzem uma estimativa precisa do coeficiente de retroespalhamento em áreas homogêneas (estacionárias), além de preservar as bordas e as estruturas texturais em cenas não estacionárias. Também adaptam alterações nas propriedades locais do retroespalhamento de terreno e modelam o speckle como sendo estacionário, porém, o sinal do alvo pode não ser estacionário, pois o retroespalhamento médio muda de acordo com o tipo de alvo. São exemplos desses filtros os de Frost, Lee, Map Gama e média local.

Para efeito de avaliação dos filtros, serão calculadas as médias normalizadas de regiões homogêneas, cujo cálculo consiste na divisão da média da imagem filtrada pela média da imagem original. Quanto mais homogênea for a região, mais próximo de 1 será o resultado.

Outra medida para avaliação dos filtros é o desvio padrão da média, calculado pela divisão do desvio-padrão pela média. Essa medida determina a habilidade do filtro em reduzir o speckle em uma região homogênea. Quanto menor o valor do desvio padrão da média, maior a capacidade de redução do speckle.

\section{Correção geométrica}

Uma imagem bruta usualmente apresenta distorções geométricas significativas, razão pela qual não pode ser utilizada com finalidade cartográfica antes que sejam aplicadas as devidas correções. Existem várias fontes de distorção como variação de altitude, atitude e velocidade do sensor. 0 propósito da correção geométrica é compensar a distorção introduzida por esses fatores e dar à imagem a integridade geométrica de um mapa (LILLESAND, 1994).

Os modelos para correção geométrica podem ser físicos ou generalizados. Nos modelos físicos, cada parâmetro tem um significado físico e tem relação com a posição e a orientação do sensor, com relação a um sistema de coordenadas do espaço-objeto. Nos modelos generalizados, a transformação entre os espaços, imagem e objeto é representada como uma função geral como, por exemplo, um polinômio. Em geral, por não precisarem de conhecimento da geometria do sensor, os modelos generalizados podem ser aplicados a diferentes tipos de sensores (ARAKI, 2005).

Os parâmetros da transformação geométrica são calculados utilizando-se o método dos mínimos quadrados (MMQ) para um conjunto de pontos conhecidos nos dois espaços, imagem e terreno, denominados de pontos de controle. Um modelo utilizado na correção geométrica de imagens de alta resolução espacial é o de polinômios racionais, cuja equação geral é demonstrada abaixo (Equação 2). 
Onde Ln é a coordenada de imagem linha e Cn é a coordenada de imagem coluna normalizadas, correspondentes a um ponto objeto cujas coordenadas normalizadas são Xn, Yn, e Zn.

A relação entre as coordenadas do mosaico de ortofotos e MDT $(E, N, H)$ e da imagem-radar $\left(E^{\prime}, N^{\prime}\right)$ pode ser descrita geometricamente por uma transformação projetiva que, matricialmente, se apresenta na equação a seguir (Equação 3).

$$
\left(\begin{array}{l}
E^{\prime} \\
N^{\prime} \\
0 \\
1
\end{array}\right)=\left(\begin{array}{llll}
a_{11} & a_{12} & a_{13} & a_{14} \\
a_{21} & a_{22} & a_{23} & a_{24} \\
a_{31} & a_{32} & a_{33} & a_{34} \\
a_{41} & a_{42} & a_{43} & a_{44}
\end{array}\right)\left(\begin{array}{c}
E \\
N \\
H \\
1
\end{array}\right)
$$

Para correção da imagem RADARSAT-2, será aplicada a transformação projetiva, que relaciona coordenadas tridimensionais de um objeto com a sua representação no espaço bidimensional. Para isso, foram coletadas em campo as coordenadas de pontos de controle bem definidos na imagem e distribuídos de maneira homogênea.

A imagem GeoEye passou pelo processo de correção geométrica conhecido como registro, realizado para compatibilizar duas imagens diferentes de uma mesma área. Nesse processo, uma imagem é tomada como referência e as demais são corrigidas com base no sistema de referência da imagem adotada. Para o registro, foi utilizada a técnica imagem-mapa e, como referência, o mapa de arruamento fornecido pelo IPPUC.

\section{Segmentação da imagem}

A segmentação de imagem é um processo que visa dividir a imagem em regiões com aspecto semelhante (alguns objetos de interesse em uma imagem), podendo isolar os pixels que não fazem parte desses objetos.

A segmentação mean shift, desenvolvida por SONKA, é uma técnica que agrupa por região as cores semelhantes, estatisticamente diminuindo a quantidade total de cores na imagem. É um procedimento para localizar os máximos de uma função densidade a partir de dados discretos amostrados dessa função (MACHADO, 2006).

O software Edge Detection and Image Segmentation System (EDISON) é um programa disponibilizado pela Universidade de New Jersey, onde está implementada a técnica mean shift. Nesse software, as imagens são segmentadas com base na cor e na quantidade de pixels. Cada segmento é colorido com a cor média dos pixels que a constituem, sendo possível definir o tamanho máximo de cada segmento bem como a distância a partir da qual duas cores são consideradas distintas. (PIMENTA 2008).

Assim, para auxiliar na extração de informações da imagem GeoEye, será realizada a segmentação, a fim de reduzir o volume de dados e obter apenas as características significativas da imagem.

\section{Classificação por Árvores de Decisão}

As Árvores de Decisão são mecanismos de categorização de dados por meio de divisões hierárquicas, com o objetivo de facilitar o entendimento das relações entre as variáveis envolvidas. As variáveis em estudo são rotuladas e representadas em forma de árvore invertida, separando os diferentes níveis de classificação em níveis hierárquicos (ARAKI, 2005). 
Um classificador hierárquico é um algoritmo para rotular padrões desconhecidos, usando uma sequência de decisões. A Árvore de Decisão é composta por um nó raiz, um conjunto de nós interiores e os nós terminais, chamados folhas. Os nós terminais representam a classificação final. 0 processo de classificação é formado por um conjunto de regras que determinam o caminho a ser seguido, começando do nó raiz até chegar às folhas. A natureza das decisões é definida pela sequência de atributos que ocorrem dentro de uma árvore, o que afetará o resultado da classificação. Assim, sabe-se que a eficiência e o desempenho dessa abordagem são fortemente afetados pelo algoritmo para indução adotado. A Figura 2 ilustra um exemplo simples de Árvore de Decisão, usando como valor de entrada a refletância de pixels da imagem a ser classificada em árvore, arbusto, pântano ou água.

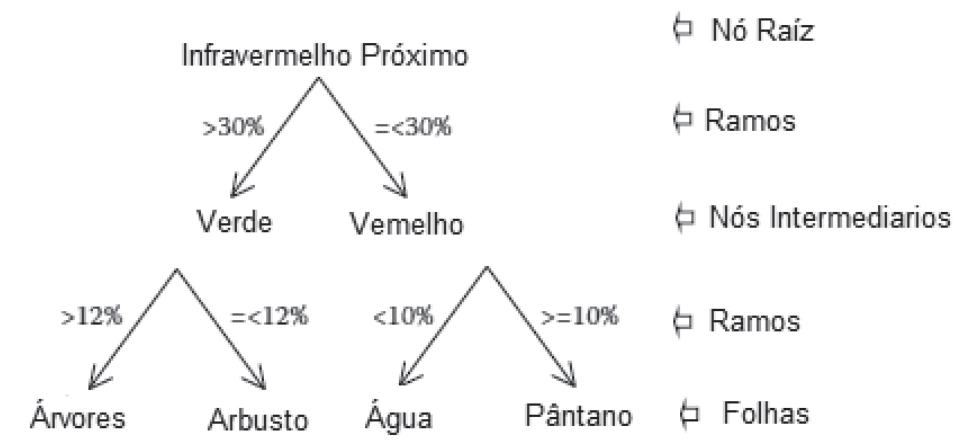

Figura 2. Árvore de decisão.

Fonte: Adaptado de Tso (2001).

Existem duas maneiras de se criar uma Árvore de Decisão, manual ou automaticamente. A abordagem manual é baseada no conhecimento do usuário e pode gerar resultados insatisfatórios quando se tem um grande volume de dados para analisar (MACHADO, 2006).

Com o grande interesse no estudo de métodos automáticos de criação de Árvores de Decisão com classificadores como ID3 (Iterative Dichotomizer 3) e C4.5, por exemplo, têm crescido nos últimos anos pesquisas relacionadas ao assunto, com desempenhos promissores.

0 método de indução de decisão ID3 foi proposto por Quinlan em 1979, que observou que a construção de uma árvore de decisão requer estratégias de "divisão e conquista" por meio de divisão recursiva de dados para gerar pequenas árvores.

Para um conjunto de várias instâncias $S$ e um conjunto de $n$ classes $C=\{C 1, \ldots, C n\}$, sendo pi a proporção de instâncias no conjunto $S$ pertencentes à classe i, a entropia do conjunto $S$ é expressa como se apresenta na Equação 4:

$$
\operatorname{Entropia}(S)=\underset{i=1}{-\sum_{i}^{n}} p_{i} \log _{2}\left(p_{i}\right)
$$

A entropia atinge valor máximo igual a $\log 2 \mathrm{n}$, quando $\mathrm{p} 1=\mathrm{p} 2=\ldots=\mathrm{pn}=1 / \mathrm{n}$, expressando a existência de homogeneidade. A medida de ganho de informação tem relação com a redução esperada na entropia se as instâncias no conjunto $S$ forem separadas com um determinado atributo $A$ das instâncias de $\mathrm{S}$, com Domínio $(\mathrm{A})=\{\mathrm{v} 1, \mathrm{v} 2, \ldots$, vr $\}=\mathrm{V}$. Considerando que $\mathrm{v} \in \mathrm{V}$ separa um subconjunto de elementos de $S$ e denominando esse subconjunto por Sv, a entropia desse novo conjunto é representada por Entropia(Sv). Realizando essa operação para cada elemento de $\mathrm{V}$, pode-se calcular o quanto é esperado que seja reduzida a entropia. O ganho de informação do atributo A é demonstrado pela Equação 5: 


$$
\text { Ganho }(S, A)=\text { Entropia(S) }-\sum_{V \varepsilon \vee} \frac{|S V|}{|S|} \text { Entropia(SV) }
$$

O melhor atributo é aquele que proporciona o maior ganho de informação, sendo reduzida a entropia à medida que são divididas as instâncias presentes no conjunto S. (ARAKI, 2005).

0 algoritmo ID3 não trabalha bem com os valores de atributo numérico e contínuo e não contempla a ausência ou desconhecimento de atributos, além de não fornecer aos usuários a possibilidade de poda. Esse algoritmo foi desenvolvido por Quinlan, em 1986, e uma série de melhorias no programa resultou numa versão aprimorada do algoritmo para a criação de árvore de decisão chamado C4.5. Uma dessas melhorias é conhecida como superadaptação e ocorre quando os conjuntos de treinamento utilizados são grandes; também, existe o risco de a árvore apresentar uma regularidade incoerente nos dados, segundo Luger (2004).

As melhorias incluem ainda a estratégia de poda da árvore, que, nesse contexto, significa reduzir algumas subárvores a folhas, ou, de outra forma, um ramo da árvore, a partir de determinado nó, é cortado (transformado em folha). 0 corte de um ramo da árvore é guiado por um teste estatístico que considera os erros em um nó e a soma dos erros nos nós que descendem desse nó. Além de permitir o tratamento do problema da superadaptação, o C4.5 permite lidar com valores numéricos, valores omissos e dados contendo ruído. Outra vantagem disponibilizada por esse sistema é a capacidade de validação, além da possibilidade de gerar regras de decisão a partir de árvores.

\section{Avaliação da classificação}

Um método comumente utilizado para avaliar os erros nos resultados da classificação é o da matriz de confusão. Esse método avalia a qualidade da classificação, pois apresenta erros de amostra de uma classe atribuídos a outra classe à qual ela não pertence (LILLESAND, 1994). Outra forma de avaliação consiste no índice de exatidão global, que resulta da divisão do total de pixels classificados corretamente (diagonal da matriz) pelo número total de pixels da matriz.

O índice kappa, desenvolvido por Cohen (1960), é uma medida largamente utilizada para avaliar a acurácia de uma classificação. Esse índice reflete a proporção de acerto após a eliminação do acerto por acaso e é calculado a partir da matriz de confusão, com base na diferença entre concordância observada (diagonal principal da matriz de confusão com a exatidão global) e a chance de concordância entre os dados de referência e uma classificação aleatória (produto entre os totais marginais da matriz).

Segundo Pinto (2010), a análise dos resultados do índice kappa segue as indicações expressas pelo Quadro 1, onde se têm intervalos de valores e a respectiva concordância. Quanto mais próximo de 1 o valor de kappa, mais acurada está a classificação.

\begin{tabular}{|c|c|}
\hline Valor de kappa & Concordância \\
\hline 0 & Pobre \\
\hline $0-0,20$ & Ligeira \\
\hline $0,21-0,40$ & Considerável \\
\hline $0,41-0,60$ & Moderada \\
\hline $0,61-0,80$ & Substancial \\
\hline $0,81-1$ & Excelente \\
\hline
\end{tabular}

Quadro 1. Valores de referência na análise kappa.

Fonte: PINTO (2010). 
Serão analisados como forma de avaliação da classificação tanto a matriz de confusão quanto o índice Kappa.

\section{Resultados e discussões}

\section{Calibração}

O primeiro procedimento realizado antes da utilização de uma imagem radar é a calibração. A Figura 3 apresenta a matriz de calibração, em que se tem maior intensidade de correção (região mais clara) quanto mais distante do sensor estiver o alvo e menor intensidade de correção (mais escura) quanto mais próximo do sensor estiver o alvo.

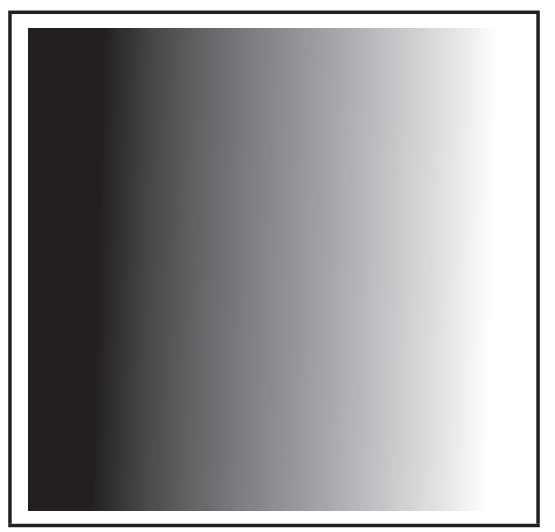

Figura 3. Matriz de calibração Fonte: Elaboração própria.

A Figura 4-a apresenta um recorte da imagem e a 4-b, o resultado da calibração correspondente.

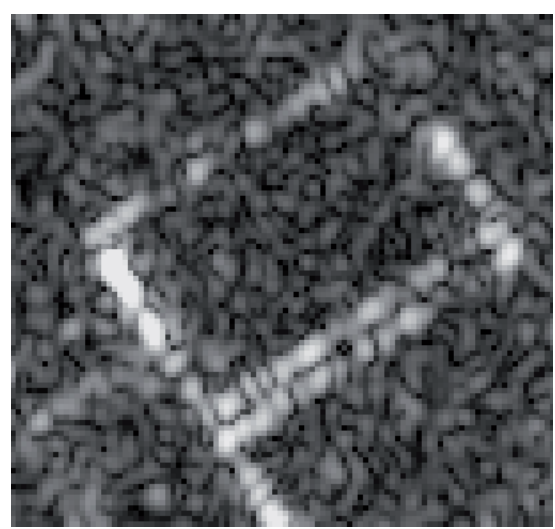

4-a) Imagem original

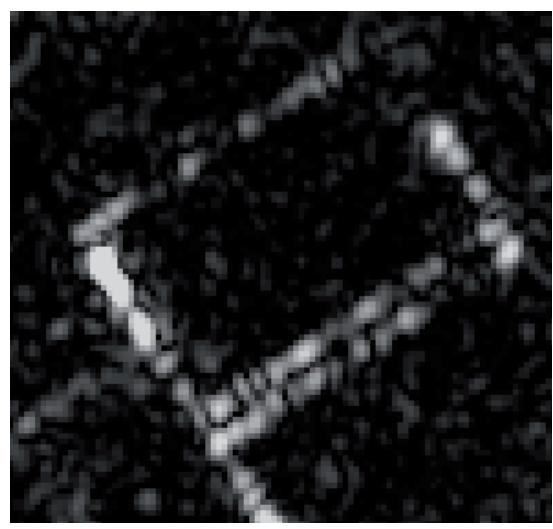

4-b) Imagem calibrada

Figura 4. Calibração da Imagem Radar.

Fonte: Elaboração própria.

Pode-se observar que a imagem calibrada apresenta a mesma feição com maior nitidez do que a imagem original. 


\section{Filtragem}

Após a calibração, foi feita a filtragem para redução do speckle. Foram aplicados o filtro Linear com janelas $3 \times 3$ e $5 \times 5$, o filtro Lee e o filtro Frost, com janelas $3 \times 3$. Os filtros foram implementados no software Matlab para possibilitar o processamento; os valores de pixel da imagem em decibéis sofreram a aplicação de um fator de escala. 0 resultado da filtragem é apresentado na Figura 5.

\section{Imagem RADAR original}

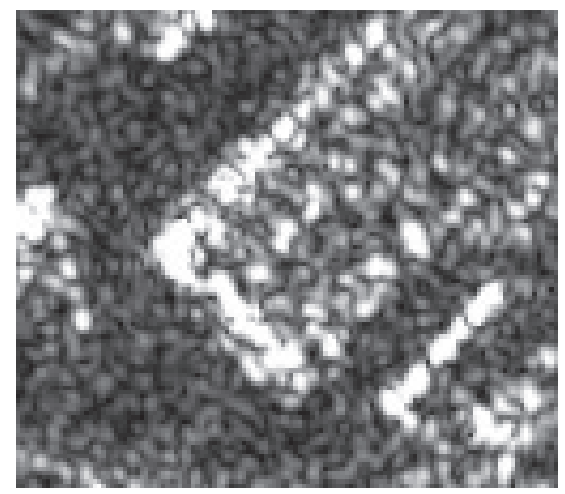

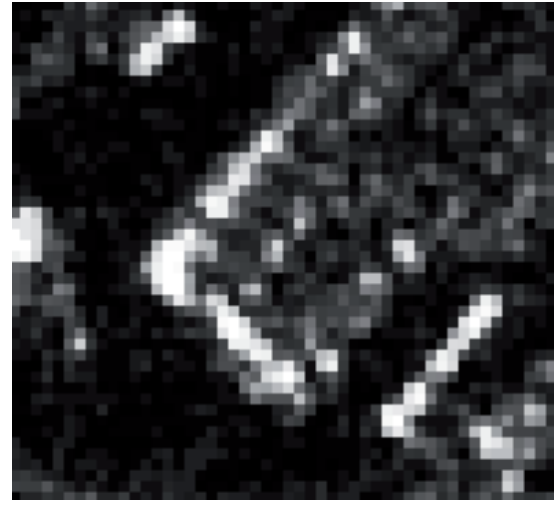

5-a) Filtro linear com janela $3 \times 3$

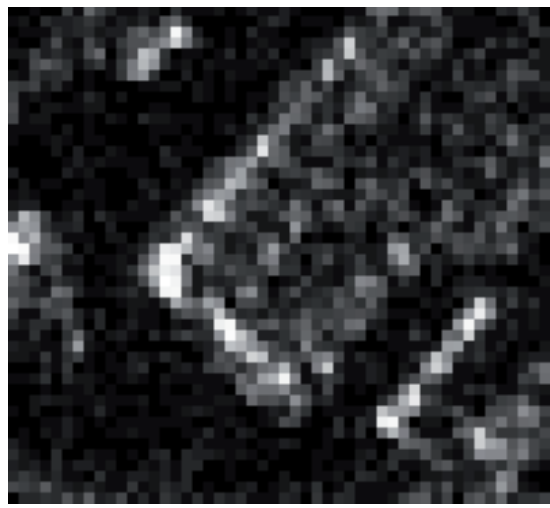

5-c) Filtro Lee

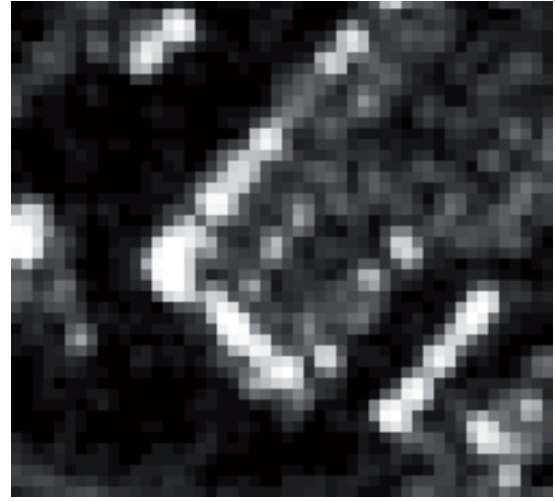

5-b) Filtro linear com janela 5x5

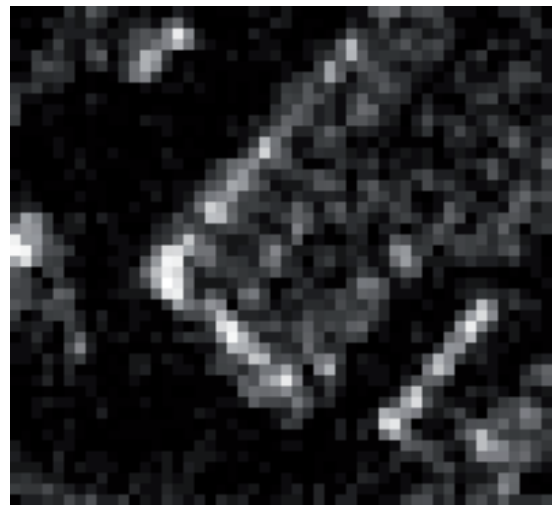

5-d) Filtro Frost

Figura 5. Imagens filtradas.

Fonte: Elaboração própria.

O recorte apresentado na Figura 5 corresponde a uma edificação cuja lateral é recoberta por material metálico. 0 filtro linear de máscara 3x3, como ilustra a Figura 5-a, retirou parte do ruído e não modificou a aparência da feição original. Já com a máscara $5 \times 5$ (Figura 5-b), ocorre um realce 
dos contornos do objeto. O mesmo não ocorre com os filtros adaptativos, Lee e Frost "figuras 5-c e 5-d", que permaneceram com ruído e apresentaram desvanecimento das linhas da feição imageada.

A aplicação dos filtros resultou em alterações na estatística da imagem, considerando os valores de média e desvio padrão, como se observa no Quadro 2.

\begin{tabular}{|c|c|c|}
\hline Imagem & Média & Desvio-Padrão \\
\hline Original & 602.4 & 2252.2 \\
\hline Filtro linear 3×3 & 602.3 & 2003.6 \\
\hline Filtro linear 5×5 & 438.0 & 1232.8 \\
\hline Filtro Lee & 588.4 & 2131.5 \\
\hline Filtro Frost & 583.0 & 1991.0 \\
\hline
\end{tabular}

Quadro 2. Estatística dos filtros das imagens.

Fonte: Elaboração própria.

Analisando o desvio-padrão e a média, percebe-se que o filtro com maior delimitação do speckle foi o filtro linear de janela $5 \times 5$. Esse, então, será o filtro adotado para as próximas etapas, uma vez que não há necessidade de preservação de áreas homogêneas quando se analisa uma cena urbana.

\section{Correção Geométrica}

Após a filtragem, foi realizada a correção geométrica utilizando o modelo de transformação projetiva, sendo coletados pontos homólogos na imagem RADAR e no mosaico. Os pontos coletados possuem coordenadas planas do mosaico de ortofotos e informação altimétrica oriunda do modelo digital de terreno, gerado a partir das curvas de nível. No total, foram coletados 20 pontos, distribuídos na imagem de forma a abranger toda a área; 4 foram descartados por apresentarem discrepância em relação ao conjunto. Os pontos foram separados em dois conjuntos de pontos, um para a determinação dos parâmetros de transformação, com 10 pontos, e outro para a verificação, com 6 pontos. Estão apresentados no Quadro 3.

\begin{tabular}{|c|c|c|c|}
\hline Pontos & Mínimo & Médio & Máximo \\
\hline Pontos de controle & 0,186591 & 0,921747 & 1,313599 \\
\hline Pontos de verificação & 0,352357 & 0,988298 & 2,191417 \\
\hline
\end{tabular}

Quadro 3. Valores dos resíduos para os pontos de controle de verificação.

Fonte: Elaboração própria.

A imagem resultante da transformação apresentou bom resultado visual, principalmente na região do Bairro Alto (Figura 6), que aparecia com ondulações na imagem original (Figura 6-a) em decorrência da grande variação do relevo no local. Já na Figura 6-b, nota-se que as ruas foram retificadas. 


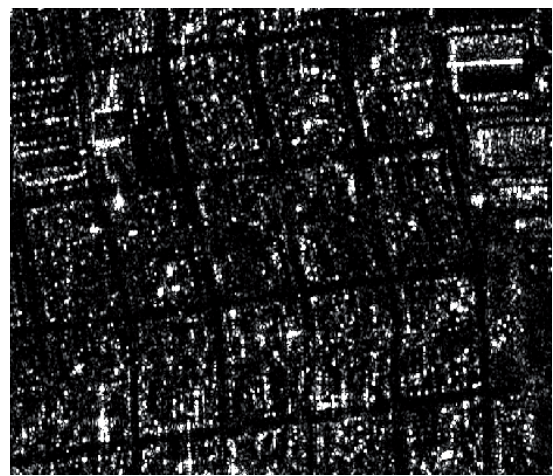

6-a) Imagem original

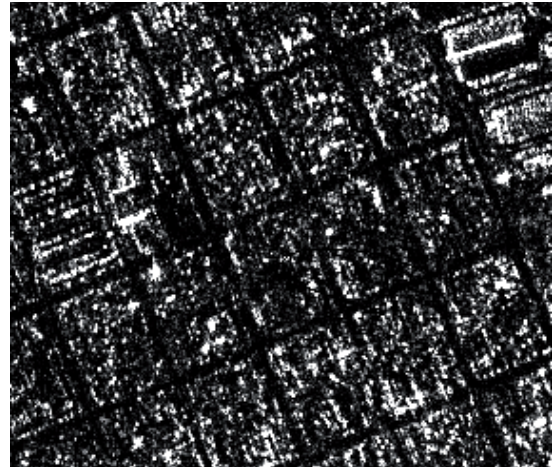

6-b) Imagem corrigida

Figura 6. Correção Geométrica

Fonte: Elaboração própria.

Os resíduos encontrados, tanto para o experimento utilizando as coordenadas do mosaico de ortofotos quanto para o experimento com as coordenadas levantadas em campo, foi inferior a um pixel.

\section{Classificação das imagens}

Tendo conhecimento da realidade de campo de algumas edificações, foram coletadas amostras de tipos de telhado nas imagens. As classes de telhado selecionadas foram: fibrocimento, telhado de cerâmica, telha pintada ou laje e telha metálica. Além dos telhados, foram selecionadas amostras das classes asfalto, solo e vegetação. Foram realizados quatro experimentos, classificando as imagens pelo método pixel a pixel: o primeiro com as bandas originais da imagem GeoEye, o segundo com a imagem GeoEye combinada com a imagem-radar, o terceiro com a imagem GeoEye segmentada e o último com a imagem segmentada combinada com a imagem-radar.

A segmentação da imagem foi realizada no software EDISON e foram testados os parâmetros de cor, forma e região. Quando se atribui maior peso para cor, ocorre a homogeneização das feições, englobando feições de aparência espectral semelhante em uma mesma classe. A segmentação que apresentou melhor resultado foi a com peso 9 para forma, 6.5 para cor e região mínima de 15 pixels.

A partir das amostras, foi gerada a árvore de decisão que criou as regras para a classificação. Essas regras analisam o valor de pixel em determinada banda e, dependendo do valor, atribui-Ihe uma classe ou parte-se para a análise do valor do mesmo pixel em outra banda, e assim sucessivamente, até que todos os pixels tenham uma classe correspondente.

Foi realizada a avaliação da classificação utilizando o índice Kappa, que se refere à acurácia da classificação. Quanto mais próximo de 1 o valor de kappa, maior a proporção de acerto da classificação, conforme se mostra no Quadro 4.

\begin{tabular}{|c|c|c|}
\hline Imagem utilizada no experimento & KAPPA & VARIÂNCIA \\
\hline GEOEYE & 0,836466 & $9,58 \mathrm{E}-05$ \\
\hline GEOEYE+RADAR & 0,904561 & $6,11 \mathrm{E}-05$ \\
\hline GEOEYE Segmentada & 0,988425 & $7,81 \mathrm{E}-06$ \\
\hline GEOEYE Segmentada+RADAR & 0,993195 & $4,6 \mathrm{E}-06$ \\
\hline
\end{tabular}

Quadro 4. Valores do indice kappa da classificação.

Fonte: Elaboração própria. 
Podemos observar que os experimentos realizados com as imagens combinadas apresentaram maior valor para o índice kappa. O melhor resultado aparece quando se tem a combinação da imagem GeoEye segmentada com a imagem RADARSAT-2. O resultado final das classificações é apresentado na Figura 7.

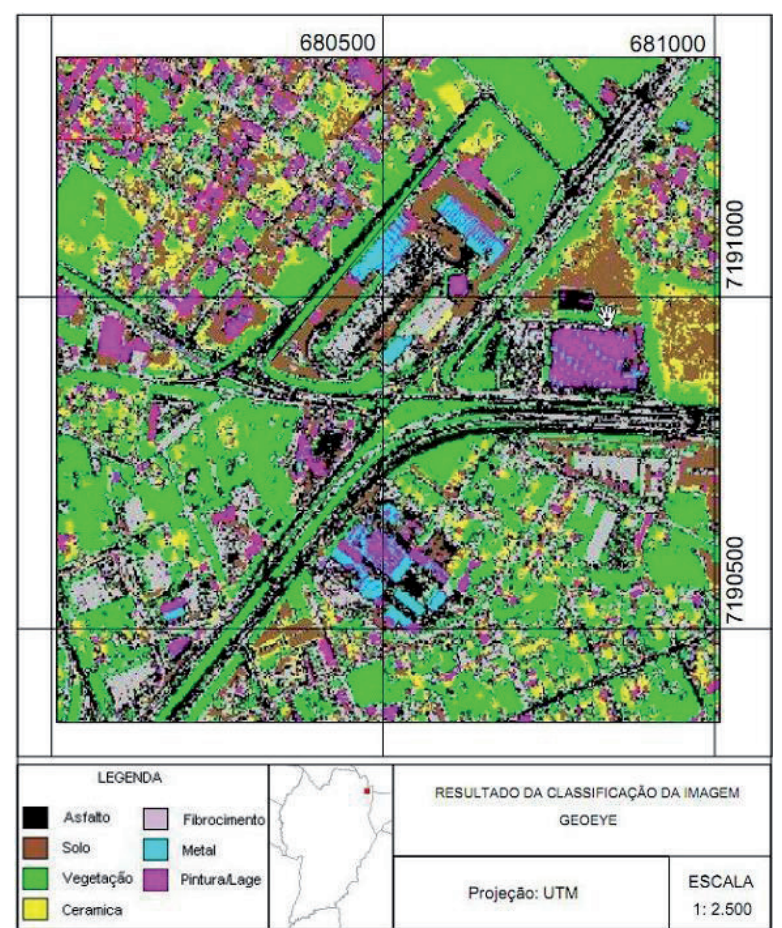

7-a) Classificação da imagem GeoEye

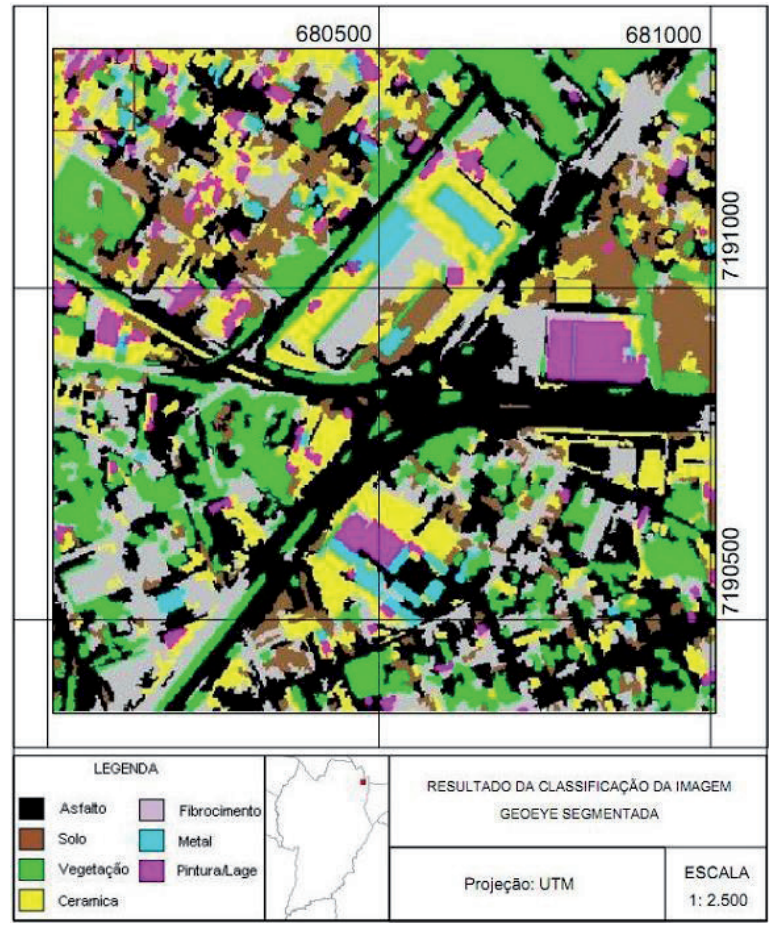

7-c) Classificação da imagem segmentada

Figura 7. Classificação Final

Fonte: Elaboração própria.

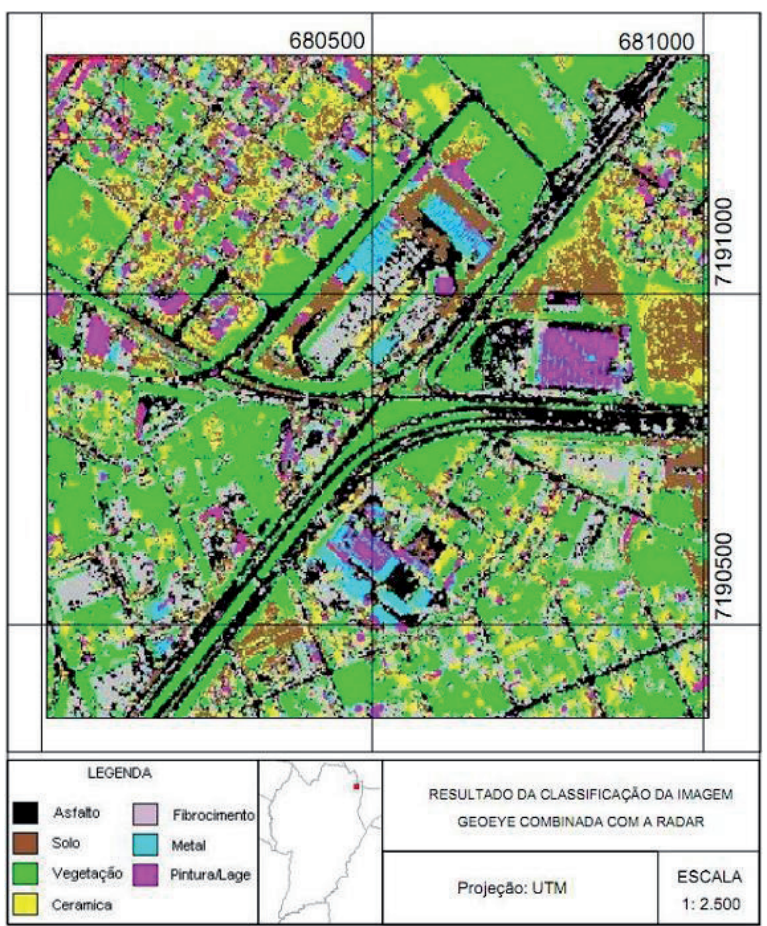

7-b) Classificação da imagem GeoEye combinada com a imagem RADARSAT - 2

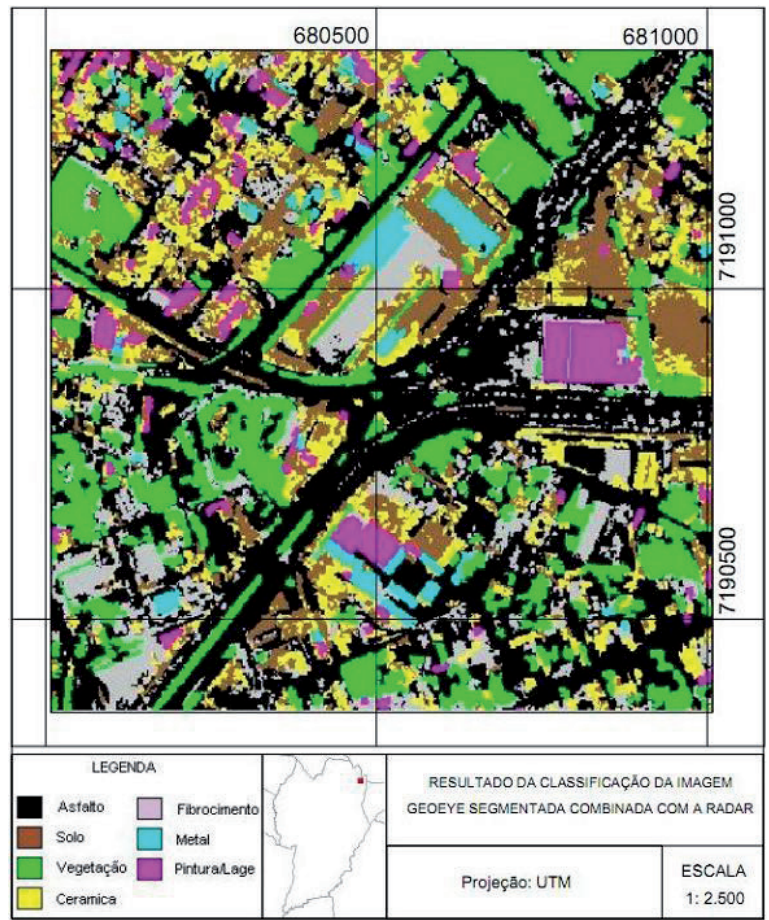

7-d) Classificação da imagem segmentada combinada com a imagem RADARSAT-2 
Quando se utilizou a imagem GeoEye segmentada, houve menor confusão entre as classes, como se pode observar nas figuras 7-c e 7-d. Nesses experimentos, as classes estão melhor discriminadas, o que é demonstrado tanto visualmente quanto na análise dos valores do índice kappa.

A classificação da imagem segmentada, combinada com a imagem-radar, foi a que apresentou menor confusão, dado perceptível principalmente entre as classes telhado de cerâmica e solo. A principal contribuição da imagem RADARSAT no resultado final se dá na caracterização de estruturas metálicas que apresentam alto fator de reflexão nessa imagem.

Comparando o resultado da classificação com a realidade de campo da região, pode-se comprovar que o método experimentado permitiu a separação das classes de interesse. As amostras coletadas em campo foram corretamente classificadas no experimento que reuniu a informação da imagem GeoEye segmentada com a imagem RADARSAT-2.

\section{Conclusão}

O resultado dos experimentos de classificação utilizando árvore de decisão apresentou-se coerente com a realidade da área de estudo. 0 melhor resultado foi verificado no experimento utilizando a imagem GeoEye segmentada combinada com a imagem-radar, uma vez que essa imagem contribui para a caracterização de estruturas metálicas que apresentam alto fator de reflexão. 0 valor do índice kappa ficou próximo de 1 para as classes analisadas neste experimento. Foi possível ainda comprovar a confiabilidade da técnica por meio da visita de campo.

Porém, essa técnica depende da habilidade do analista na aquisição de amostras representativas de cada classe para a construção da árvore de decisão. Algumas classes tiveram resultados mais acurados, enquanto outras apresentaram confusão. Áreas com menor semelhança espectral ficaram bem definidas, já as áreas semelhantes não repetiram o resultado.

Recomenda-se que, em estudos futuros, sejam realizados experimentos utilizando outras técnicas de classificação, como, por exemplo, a lógica fuzzy. Outra possibilidade é a geração de texturas para ajudar na discriminação dos objetos. Recomenda-se ainda realizar estudos comparativos entre as diferentes formas de polarização, o que não foi possível neste trabalho, visto que só havia uma imagem disponível.

\section{Information from urban scene matching RADARSAT-2 and GeoEye images}

\section{Abstract}

This research analyzed RADARSAT-2 image contribution to discriminate elements of an urban scene, when fused to a GeoEye image. The advantage of radar image is their independence from weather conditions and the nominal spatial resolution in ultrafine mode is 3 meters. The multispectral images, even high-resolution doesn't discriminate all the elements that make up an urban scene. The main objective was to discriminate elements of the scene using the combination of information from optical imaging and radar and also check if the projective transformation model is suitable for geometric correction of the picture. Moreover, analyze the effect of filters to reduce speckle in the image and find the radar image contribution to identify elements present in the scene. The projective transformation showed a satisfactory result in image correction, although the angle of sight was lateral, values 
of the control and check points were smaller than 1 meter. In order to treat speckle, the $5 \times 5$ window statistical average filter was the one that allowed the removal of speckle with greater efficiency. The results of classification experiments using decision tree were consistent to the reality of the study area. The best result was observed in the experiment using the GeoEye segmented image combined with the radar one, as this contributes to metal structures characterization that has a high reflection factor. It was possible to prove the technical reliability through the field visit.

Keywords: GeoEye. Speckle. Radar image. Image classification.

\section{Referências}

ARAKI, H. Fusão de informações espectrais, altimétricas e de dados auxiliares na classificação de imagens de alta resolução espacial. 2005. Tese (Doutorado em Ciência da Terra) - Universidade Federal do Paraná, Setor de Ciências da Terra, Curitiba, 2005.

CCRS (CANADA CENTRE FOR REMOTE SENSING). Disponível em: < http://www.ccrs.nrcan.gc. ca/>. Acesso em: 4 ago. 2008.

COHEN, J. A coefficient of an agreement for nominal scales. Educational and Psychological Measurement, n. 20, p. 37-46, 1960.

GEOEYE Product Guide v1.0.1. Disponível em: <http://www.Geoeye.com/CorpSite/>. Acesso em: 4 out. 2010.

INPE (Instituto Nacional de Pesquisas Especiais). Disponível em: <http://www.cbers.inpe.br/?content=introducao $>$. Acesso em: 10 jun. 2009.

LEEUW, M. R.; CARVALHO, L. M. T. Performance evaluation of several adaptative speckle filters for SAR imaging. In: SIMPÓSIO BRASILEIRO DE SENSORIAMENTO REMOTO, 14, 2009. Anais... Natal, Brasil, 2009, p. 7299-7305.

LILLESAND, T.; KIEFER R.W. Remote Sensing and Image Interpretation. Wiley. 3. ed. New York, 1994.

LUGER, G. F. Inteligência artificial: estruturas e estratégias para a resolução de problemas complexos. Porto Alegre: Bookman, 2004. 774p.

MACHADO, A. M. L. Extração automática de contornos de edificações utilizando imagem gerada por câmara digital de pequeno formato e dados LIDAR. 2006. Tese (Doutorado em Ciências da Terra) - Universidade Federal do Paraná, Setor de Ciências da Terra. Curitiba, 2006.

MOLCH, K. Radar earth observation imagery for urban area characterization. JRC Scientific and Technical Reports, 2009.

PIMENTA, N. R. de C. PhotoFinder 2: classificação e pesquisa de fotografias digitais. 2008. Dissertação (Mestrado) - Universidade Técnica de Lisboa, Instituto Superior Técnico. Lisboa, 2008. 
PINTO, J. S. Lopes J.M., Oliveira J.V., Amaro J.P., Costa L.D. Métodos para Estimação de Reprodutividade de Medidas. Disponível em: < http://users.med.up.pt/joakim/intromed/web_t9_g1.htm>. Acesso em 10 jan. 2011.

SOARES, J. V. Fundamentos de Radar. Disponível em: <http://www.dsr.inpe.br/dsr/vianei/radar/ DOCs_PDFs/ > . Acesso em: 10 out. 2010.

TSO, B.; MATHER, P. M. Classification Methods for Remotely Sensed Data. 2. ed. Londres: Taylor \& Francis Group, 2001.

UFPR, Orientação para Normalização de Trabalhos Acadêmicos. Disponível em: <http://www.portal.ufpr.br/tutoriais_normaliza/modelo_dissertacao.pdf> Acesso em: 10 fev. 2012.

Histórico editorial

Submetido em: 05/11/2014

Aceito em: 04/03/2015 